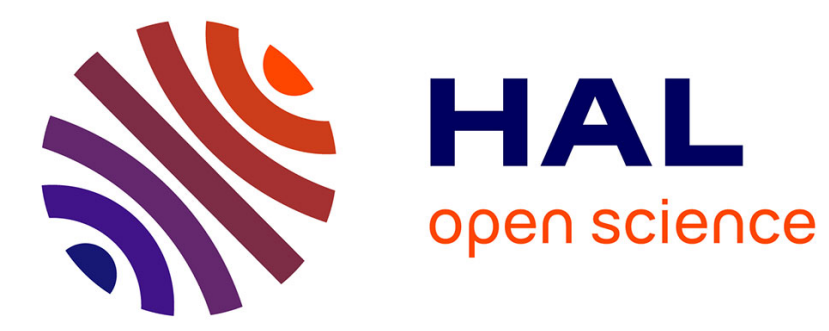

\title{
Quelques réflexions sur les contraintes spatiales et les problèmes d'échelle dans le décor des sceaux du Proche-Orient antique
}

Dominique Beyer

\section{- To cite this version:}

Dominique Beyer. Quelques réflexions sur les contraintes spatiales et les problèmes d'échelle dans le décor des sceaux du Proche-Orient antique. KTÈMA Civilisations de l'Orient, de la Grèce et de Rome antiques, 2004, 29, pp.39-49. halshs-00003817

\section{HAL Id: halshs-00003817 https://shs.hal.science/halshs-00003817}

Submitted on 4 Feb 2005

HAL is a multi-disciplinary open access archive for the deposit and dissemination of scientific research documents, whether they are published or not. The documents may come from teaching and research institutions in France or abroad, or from public or private research centers.
L'archive ouverte pluridisciplinaire HAL, est destinée au dépôt et à la diffusion de documents scientifiques de niveau recherche, publiés ou non, émanant des établissements d'enseignement et de recherche français ou étrangers, des laboratoires publics ou privés. 


\section{Extrait de KTEMA29, 2004, p. 39-49.}

\section{Quelques réflexions sur les contraintes spatiales et les problèmes d'échelle dans le décor des sceaux du Proche-Orient antique}

RÉsumé - Dans la vie quotidienne des habitants des divers pays du Proche-Orient antique, les sceaux ont occupé une place considérable. Pour leur décor, les graveurs ont été confrontés aux contraintes spatiales du support. Après l'usage de cachets circulaires ou carrés, qui remonte au Néolithique, l'invention du sceaucylindre, dont on pouvait dérouler à l'infini le décor sur l'argile, va transformer la conception de l'image. Diverses formules de traitement de l'espace disponible sont alors élaborées. De même, avec la miniaturisation des scènes, les questions d'échelle ont donné lieu à des réponses variant selon divers critères. Pour l'analyste moderne, les précieuses représentations architecturales, par exemple, présentent alors bien des difficultés d'interprétation.

ABSTRACT - In ancient Near East seals held a considerable part in the everyday life of the various peoples who inhabited the area. Cutters were much limited by the shapes of the seals when it came to engraving their designs. After the use of circular or square seals, which had started out during the Neolithic era, the invention of the cylinder seal transformed the conception of the image, as it could be rolled endlessly over clay to leave a continuous design. Several modes of space organisation were then elaborated. With the miniaturisation of scenes, scale problems were also treated in a different way and according to various criteria. When it comes to analyzing precious architectural representations, modern scholars thus face interpretation difficulties.

On ne mesure souvent pas suffisamment, en dehors du domaine des spécialistes, le fait que les sceaux du Proche-Orient ancien constituent un champ d'étude considérable, une documentation iconographique de tout premier plan, ce que l'on ne soupçonnerait guère à la seule vision, rapide et superficielle, de ces petits objets. Dans la grande plaine alluviale de Mésopotamie en particulier, la pauvreté du terroir en pierres de qualité a fait que bas-reliefs, stèles, œuvres de grand format ont été pour la plupart consacrés à la glorification des hauts-faits du souverain et réservés au développement d'un art officiel. En parallèle, en schématisant quelque peu, les autres genres, et l'iconographie religieuse en particulier, semblent s'être ainsi comme cantonnés, en toute indépendance, dans le champ réduit des sceaux ou encore dans celui des petits reliefs de terre cuite, que je ne fais qu'évoquer rapidement ici. Très tôt, le graveur de sceaux a donc été confronté au problème de la miniaturisation et a élaboré un système de codes iconographiques et symboliques susceptibles de rendre intelligible le décor des sceaux avec une remarquable économie de moyens.

Au Proche-Orient, la documentation archéologique fait pour l'instant remonter l'usage des sceaux au Néolithique, durant les VII e et VI ${ }^{e}$ millénaires. Des sites récemment explorés, tels que celui de Sabi Abyad sur la rivière Balikh, dans la haute djezireh syrienne, ont livré les empreintes, sur argile ou sur plâtre, de petits cachets circulaires, au décor gravé en creux encore bien sommaire, 
mais qui apparaissent bien comme des sceaux, destinés à sceller des contenants, et non pas simplement à servir d'amulettes. D'autres documents sont attestés sur l'Euphrate, à Buqras ou à Baghouz ${ }^{1}$. Pendant plusieurs millénaires, c'est la forme circulaire ou carrée, de petites dimensions généralement, qui est de règle. Sur les quelques $\mathrm{cm}^{2} \mathrm{du}$ champ disponible, l'image gravée en creux dans la pierre subit les contraintes du format. Ceci n'empêche pas quelques belles réalisations, comme le montrent les cachets circulaires ou parfois carrés de la fin du $\mathrm{V}^{\mathrm{e}}$ millénaire, à Tepe Gaura XIII-XI par exemple, où le décor, volontiers animalier, apparaît comme relativement fouillé, ou encore à Suse, où émerge, comme ailleurs en Iran, une iconographie d'inspiration mythologique, voisinant avec des compositions centrées très stylisées (fig. 1a-d).
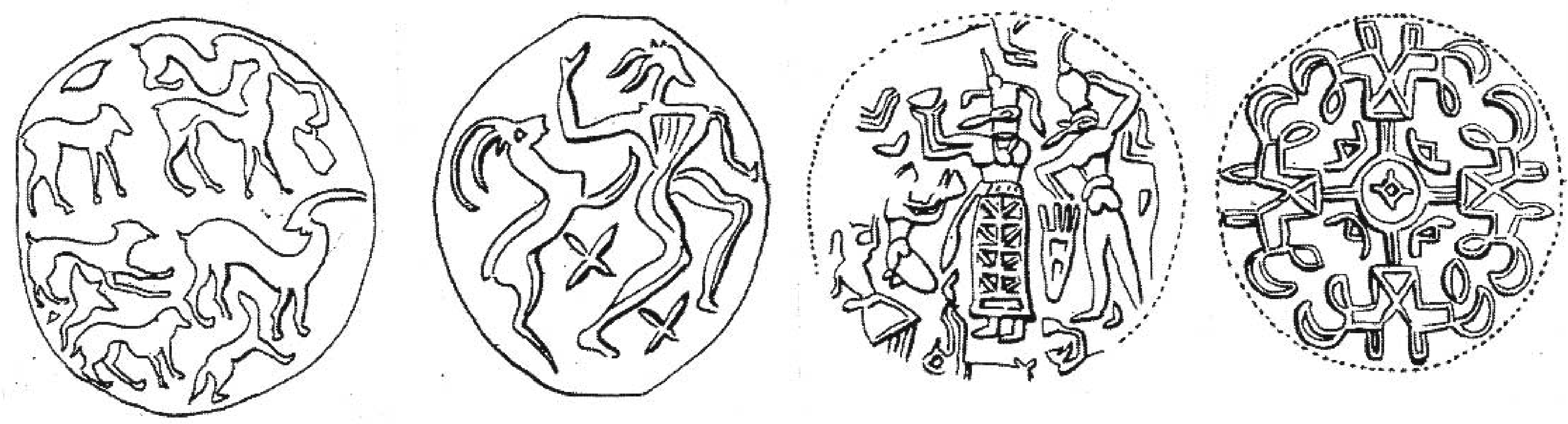

Fig. 1a-d : cachets archaïques ou leurs empreintes provenant de Tepe Gaura (a-b) et de Suse (c-d), fin du Ve millénaire av. J.-C. D'après P. AMiET, La glyptique mésopotamienne archaïque (= GMA), Paris, CNRS, 1980, nº 30, 40, 122, 129.

Mais il faut attendre l'invention du sceau en forme de cylindre pour voir se développer une iconographie plus élaborée. Cette surprenante invention, qui aura dans la plupart des pays d'Orient un succès considérable, même si certains resteront attachés à la tradition des cachets ${ }^{2}$, intervient durant cette période riche en innovations que nous appelons période d'Uruk, du nom de ce site du pays sumérien où s'élabore la première cité, au IV $V^{\mathrm{e}}$ millénaire. L'invention du sceau-cylindre, au niveau V vers 3300, y précède celle de l'écriture (Uruk IV). Quelles que soient les raisons de cette invention, le fait de donner à un sceau la forme d'un petit rouleau de pierre que l'on déroule sur l'argile des scellements de jarres, de portes ou de contenants divers, ou sur celle des tablettes cunéiformes, va profondément transformer la conception de l'image. Le décor gravé sur tout le pourtour du sceau-cylindre permet dorénavant d'imprimer dans l'argile un petit bas-relief, dont la hauteur subit obligatoirement les contraintes dimensionnelles de la matrice, mais dont la longueur peut s'étirer à l'infini. En d'autres termes, le purkullu, le graveur de sceaux, va pouvoir en partie s'affranchir des contraintes du format et créer des motifs de frises continues par répétition, lors du déroulement, des motifs gravés sur la matrice (fig. 2a-c). Les imagiers de certaines périodes ont largement utilisé ces possibilités en créant des compositions où se déroulent de savants rinceaux. Les exemples les plus caractéristiques appartiennent au style dit de Fara, dans la Mésopotamie du Dynastique Archaïque II, vers 2700-2600 (fig. 3a-b). Il s'y développe déjà abondamment l'un des thèmes majeurs de l'ensemble de la période des Dynasties Archaïques, à savoir le combat d'animaux auquel participe généralement un héros nu et des êtres hybrides, comme l'homme-taureau ou le taureau androcéphale.

(1) On pourra trouver quelques exemples reproduits dans L'Eufrate e il tempo, Le civiltà del medio Eufrate e della Gezira siriana, (Olivier Rouault et Maria-Grazia Masetti-Rouault, edd.), Milan, Electa, 1993, p. 247-250.

(2) C'est le cas, par exemple, du monde anatolien, qui est resté fidèle au cachet circulaire essentiellement, malgré la forte influence exercée par les productions de sceaux-cylindres d'Assyrie, de Mésopotamie et de Syrie du nord à l'époque des comptoirs assyriens de Cappadoce du début du second millénaire. Les Hittites de la période impériale sont ainsi restés fidèles à cette tradition, sauf dans leurs possessions de Syrie du nord. 

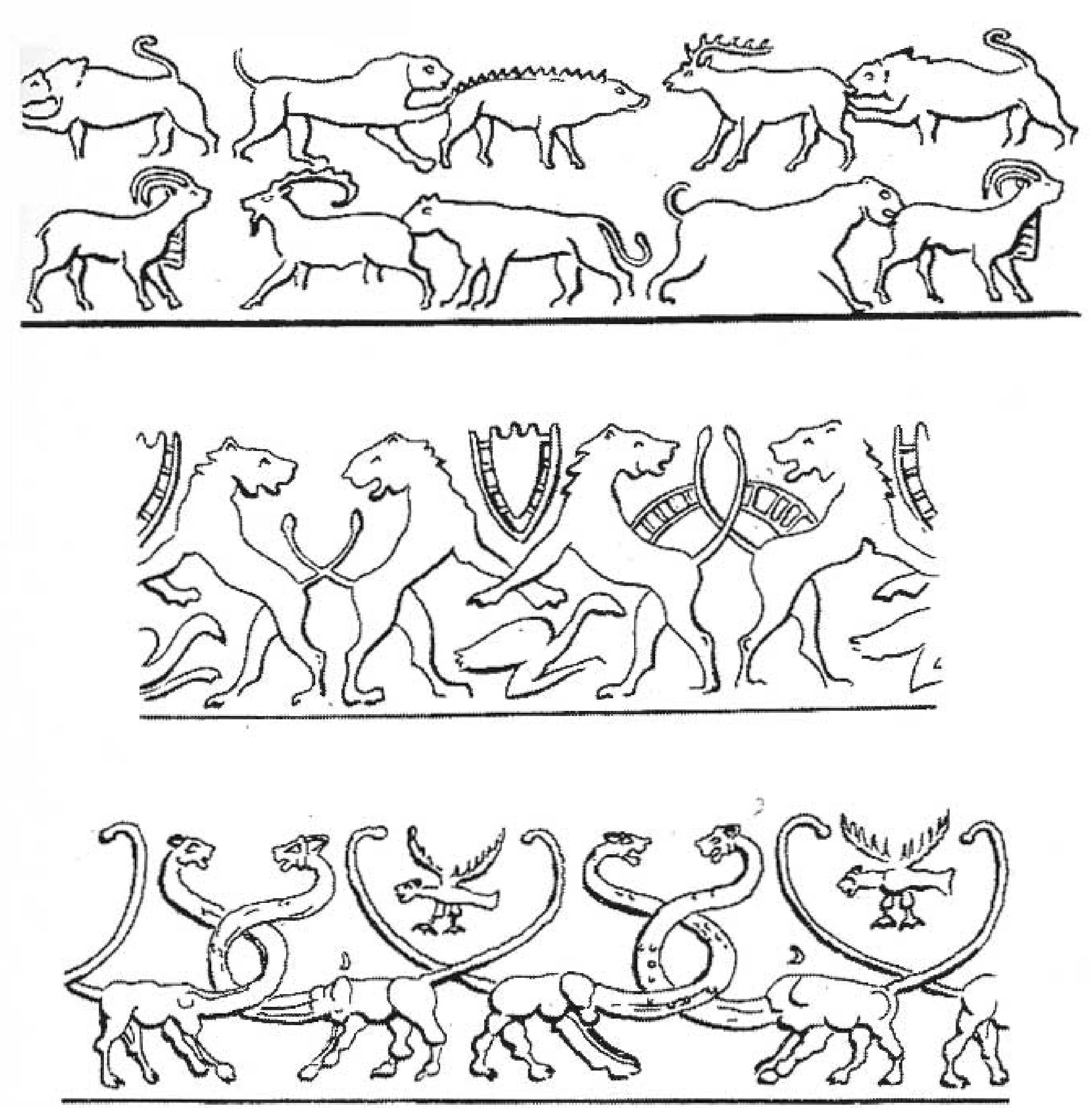

Fig. 2a-c: empreintes de sceaux-cylindres de la période proto-urbaine (IVe millénaire), déroulant des motifs de frises (GMA, $\left.\mathrm{n}^{\circ} 182,199,424\right)$
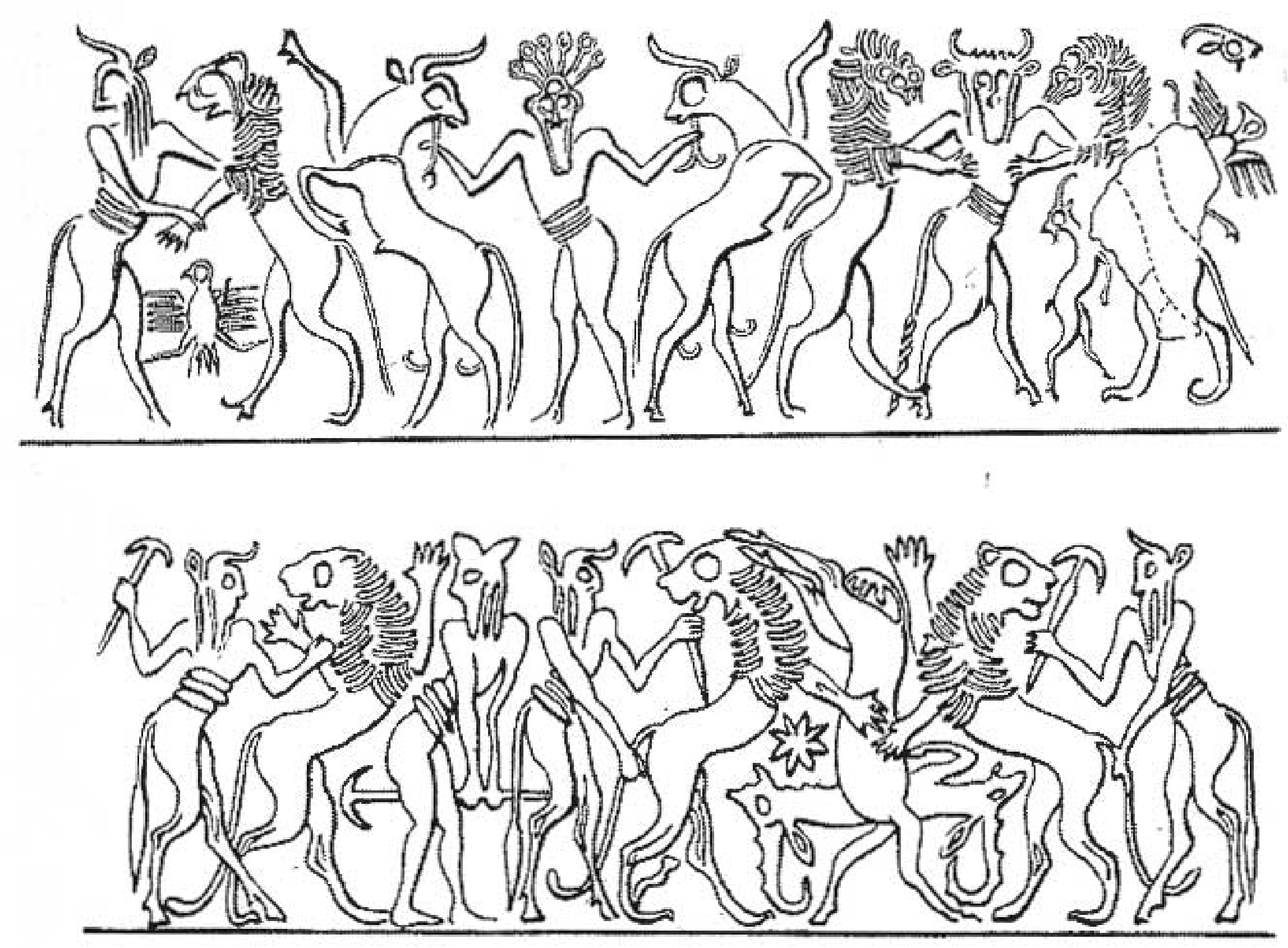

Fig. 3a-b : empreintes de sceaux-cylindres mésopotamiens du style dit de Fara, Dynastique Archaïque II, vers 2700 av. J.-C. (GMA, n 893,899$)$
Au Dynastique Archaïque III, l'animation du combat, volontiers évoquée selon un mode de composition savamment équilibré, en pyramide tronquée (fig. 4a-b), contribuant à la forte impression statique qui se dégage de ce type d'image, a fait penser à une représentation symbolique du choc des éléments constitutifs du monde, et de la stabilité de celui-ci. Aucun texte malheureusement ne vient en aide sur ce point à l'iconographe. On notera que cette thématique a été largement adoptée pendant cette période, en parallèle aux nombreuses scènes de banquet, aussi bien par les sceaux de simples fonctionnaires que par ceux des souverains à la tête des cités mésopotamiennes.

Aussi les découvertes récentes faites à Mari, dans le secteur de la porte monumentale du palais du III ${ }^{e}$ millénaire, sont-elles particulièrement importantes ${ }^{3}$. Il ne s'agit pas ici d'évoquer la problématique chronologique que ces nouveaux documents viennent alimenter, et qui touche à la chronologie de la fin des dynasties archaïques et du début de la période akkadienne, mais de souligner l'originalité de l'iconographie des sceaux royaux mis au jour et la capacité du graveur à gérer les contraintes spatiales de son support. Il s'agit de deux versions du sceau d'Ishqi-Mari (ancienne lecture Lamgi-Mari), roi de Mari bien connu par sa statuette du temple d'Ishtar retrouvée dès 1933 par André Parrot ${ }^{4}$. En deux registres superposés, dans une composition

(3) Voir D. BEYER, "Les sceaux d'Ishqi-Mari et l'idéologie royale à Mari au III millénaire ", à paraître dans les Actes du 3th International Congress on the Archaeology of the Ancient Near East, Paris, 2002.

(4) A. PARrot, Le temple d'Ishtar, Mission archéologique de Mari, I, Paris, Geuthner, 1956, p. 68-70 et pl. XXV-XXVI. 

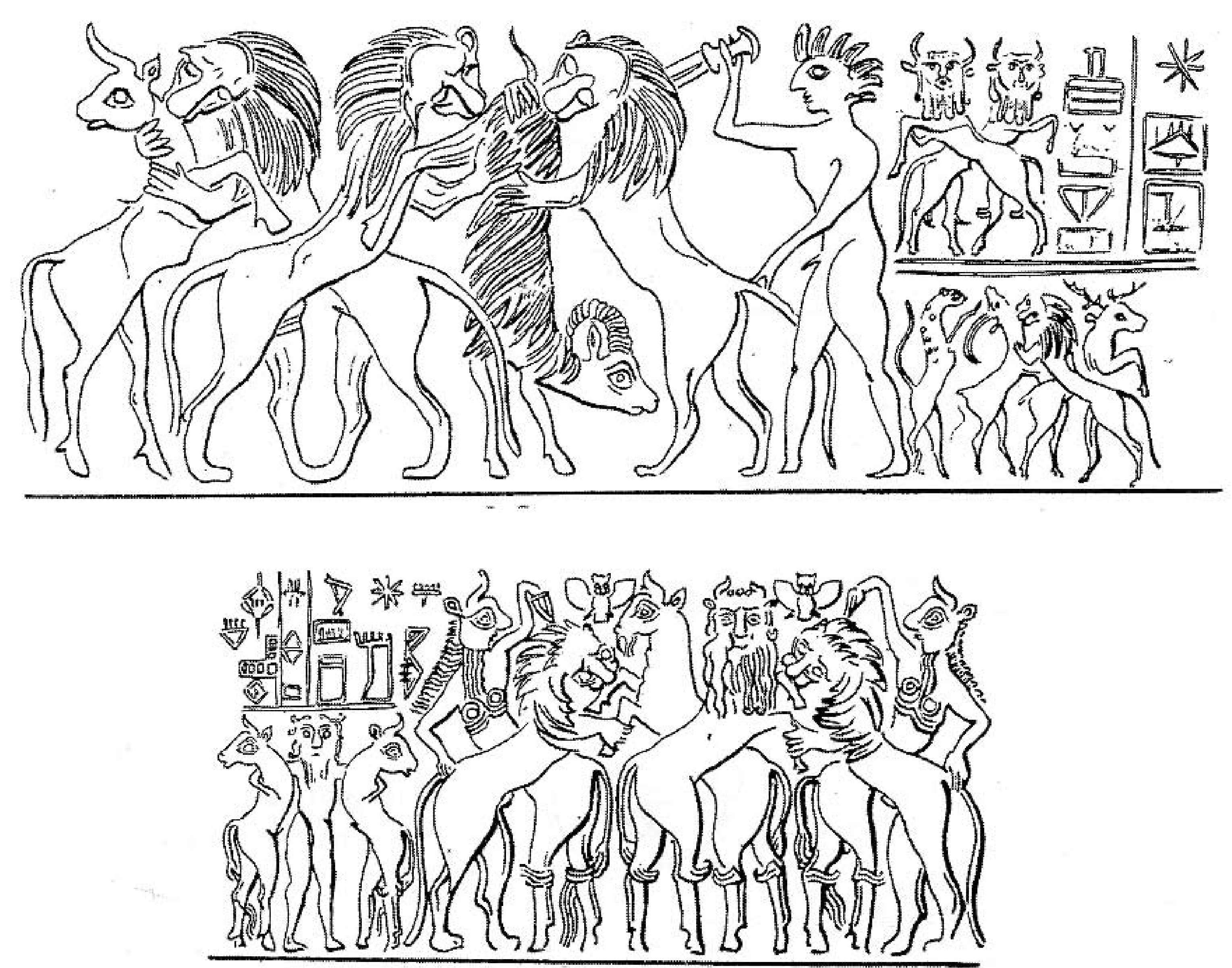

Fig. 4a-b : empreintes de sceaux-cylindres mésopotamiens du Dynastique Archaïque IIIA (a : scribe du cimetière royal d'Ur, vers 2550-2400), IIIB (b : Lugalanda de Lagash, vers 2400-2340). D’après GMA, n 1034 et 1098

très dense, on trouve sur ces sceaux ${ }^{5}$ (fig. $5 a-b$ ) les principaux codes iconographiques propres aux monuments mésopotamiens de dimensions bien plus importantes, qui célèbrent les victoires du souverain, par exemple la célèbre stèle des vautours d'Eannatum de Lagash, celle de Sargon d'Akkad ou encore l'«étendard» des tombes royales d'Ur ${ }^{6}$ : une scène de guerre figure en effet en bas, avec des fantassins culbutant leurs ennemis, dont les cadavres sont pris à partie par des rapaces, un char de guerre auquel est attaché un vaincu... En haut, c'est la célébration de la victoire royale par le banquet, scène réduite à l'essentiel: le roi trônant auquel vient apporter à boire l'échanson, portant l'habituelle cruche avec la coupe. Entre les deux, un groupe véritablement nouveau d'animaux qui ne peuvent ici que correspondre à des attributs divins: taureau d'Adad ou d'Enlil, lion portant sur son dos un prototype du lion scimmitar, symbole souvent associé, mais plus tard, à Ishtar. La déesse guerrière est également évoquée par son étoile, à moins qu'il ne s'agisse ici de l'astre solaire de Shamash, à côté du croissant lunaire, et proche des globules évoquant les Pléiades. Seraient ainsi présentes, par leurs symboles, les diverses divinités qui ont pu contribuer à la victoire royale. Tout à côté, un héros maître des animaux, domptant deux lions, est à la fois le rappel des traditions iconographiques et symboliques les plus anciennes et une allusion certaine à la victoire du souverain sur les forces hostiles (cf. aussi fig. 4b).

Il y a lieu de s'interroger sur les raisons qui ont poussé le roi Ishqi-Mari, mais, en dehors de lui, aussi un haut fonctionnaire, un shakkanakku déjà, contemporain d'un autre souverain de Mari, à adopter sur son sceau une telle iconographie belliqueuse. Il est ainsi difficile de penser à la célébration d'un événement précis. Faut-il y voir une tradition syrienne, encore insuffisamment

(5) On remarquera que le second document est la vision inversée, avec quelques modifications et simplifications, du premier. Sans doute le graveur du second sceau a-t-il copié l'image de l'empreinte sur argile du premier, qui a ainsi servi de "carton».

(6) Documents réunis par exemple chez A. PARrot, Sumer, Paris, Gallimard, 1960, p. 134-137, 146-149, 177. 

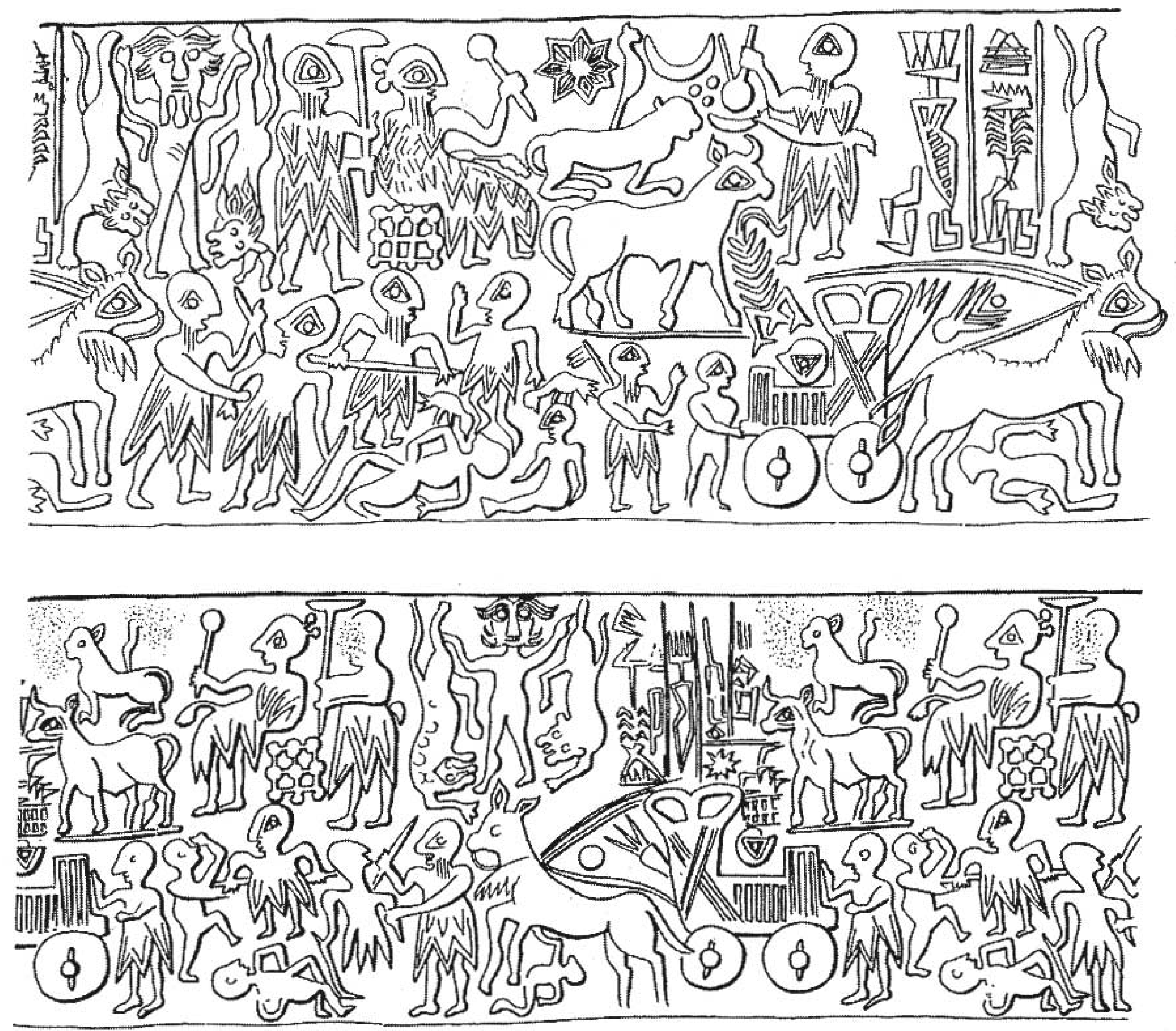

Fig. 5a-b : empreintes des deux sceaux d'Ishqi-Mari, roi de Mari, vers 2300

documentée ${ }^{7}$ ? Quoi qu'il en soit, pour notre propos, l'important est de souligner la forte originalité du sceau royal de Mari, qui réussit la prouesse de réunir, sur le champ réduit de l'image, les codes iconographiques des grands monuments de victoire de Sumer comme d'Akkad, tout en conservant l'un des thèmes favoris des sceaux, celui du maître des animaux.

Les dimensions réduites du champ disponible pour le décor du sceau ont entraîné également des problèmes d'échelle, que les graveurs ont traités diversement. D’une manière générale, le format de l'image reproduite dans l'argile, un rectangle plus ou moins allongé, se prête assez bien à la disposition paratactique des figures, à des compositions en frise, où s'impose généralement la règle de l'isocéphalie. C'est ce que montre par exemple le registre supérieur des fig. 5a-b, où le roi assis respecte cette règle, modifiant par conséquent son rapport aux autres figures. De même, lorsqu'un personnage, placé sur un socle ou un autre support, devrait apparaitre normalement plus grand que ses voisins. L'imagier a ainsi tendance à ne pas se préoccuper, dans une scène donnée, du respect de l'échelle. On peut évoquer ici un intéressant contre-exemple: le sceau de Tukulti-Ninurta I $^{\text {er }}$ montre au contraire un souci de respect des proportions relatives des différents personnages (fig. 6), qui correspond sans doute à la tendance au réalisme de l'art médio-assyrien du XIII' siècle av. J.-C. ${ }^{8}$.

(7) Certains sceaux, mais non royaux pour l'instant, originaires plutôt de Syrie du nord, semblent indiquer que ce thème belliqueux avait la faveur des imagiers à la fin du Dynastique Archaïque et au début de l'empire d'Akkad. Voir mes remarques, loc. cit. (note 3), et dans «Les sceaux de Mari au III ${ }^{e}$ millénaire: observations sur la documentation ancienne et les données nouvelles des Villes I et II ", à paraître dans Ah Purattim I.

(8) Sur ce document, cf. K. WATANABE, Die Sieglung der 'Vasallenverträge Asarhaddons' durch den Gott Assur, Baghdader Mitteilungen 16, 1985, p. 377-392. 


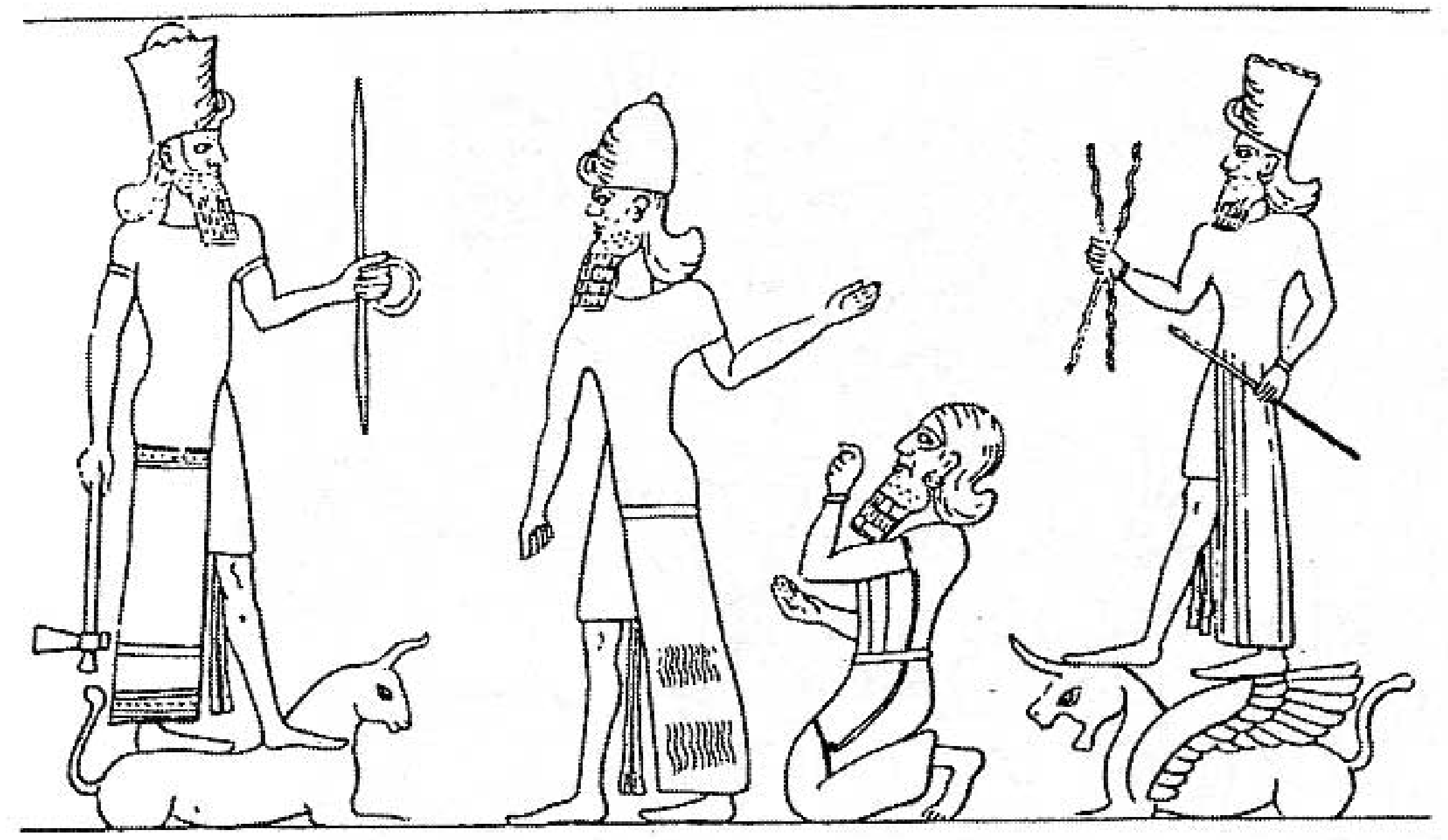

Fig. 6: empreinte du sceau attribué au roi d'Assyrie Tukulti-Ninurta Ier, $2^{e}$ moitié du XIII ${ }^{e}$ siècle av. J.-C.. D'après K. Watanabe, «Die Sieglung der 'Vasallenverträge Asarhaddons' durch den Gott Assur", Baghdader Mitteilungen 16, 1985, p. 385, fig. 4

Dans bien des cas, le personnage dominant dans une scène donnée est tout naturellement représenté de taille supérieure: sur l'empreinte proto-élamite de la fig. 7a, le «maître des animaux» apparaît alternativement sous la forme d'un taureau ou d'un lion, pouvant suggérer symboliquement l'alternance des saisons, des forces élémentaires du monde. Sur la fig. 7b, c'est le «roi-prêtre» de la période proto-urbaine qui domine de sa haute stature les matelots de cette barque cérémonielle.

On pourra s'étonner en revanche de rencontrer, dans l'art des sceaux, de nombreux témoignages d'une sorte de liberté vis-à-vis de ce que l'on pourrait appeler la hiérarchie dimensionnelle: les cortèges qui mêlent, dans des scènes de culte, divinités et orants, ces derniers le plus souvent royaux il est vrai, ne soulignent pas la différence de statut par une différence d'échelle, sauf si la divinité qui reçoit le cortège est assise, mais dans ce cas la représentation suit simplement la règle de l'isocéphalie évoquée plus haut. Plus surprenants à nos yeux sont les exemples de composition de l'image où la divinité principale est représentée de taille beaucoup plus modeste que ses acolytes: les fig. $8 \mathrm{a}$ et b montrent deux sceaux akkadiens (vers 2200 av. J.-C) illustrant les ébats des héros nus acolytes du dieu Ea, dieu de l'abîme des eaux douces de l'aps $\hat{u}$. Ce dernier, qui est pourtant leur maître, n'apparait sur ces deux documents que sous la forme d'une bien modeste silhouette d'où jaillissent les flots qui le caractérisent, même si le dieu est représenté sur la fig. $8 \mathrm{~b}$ trônant et accueillant un autre dieu venu lui rendre hommage. Dans les deux cas, ces petites images divines apparaissent comme des rappels du contexte, créant éventuellement une scène secondaire, et venant combler un espace vide de la composition. La fig. $8 \mathrm{c}$ montre un exemple similaire: sur ce sceau d'époque paléo-babylonienne, peut-être d'inspiration hurrite, c'est la figure du dieu de l'orage, avec son foudre, perché sur un dragon ailé, qui est fortement dominée par la stature des créatures étranges qui l'accompagnent. À sa droite, mais au-delà du grand personnage ailé, on trouve la figure familière du "personnage au gobelet et à la situle», considéré parfois comme l'image d'un prêtre, souvent comme celle du propriétaire du sceau ${ }^{9}$, venu ici rendre hommage au dieu de l'orage, créant ainsi une scène secondaire imbriquée dans la première.

Cette notion de scène secondaire est fréquente dans le décor des sceaux et l'imagier a souvent joué dans ce domaine sur les modifications d'échelle susceptibles de varier les modes de composition. Les sceaux des fig. $4 \mathrm{a}$ et $\mathrm{b}$ en montrent déjà des exemples à la période du Dynastique Archaïque: à côté du tableau principal, occupant toute la hauteur du champ, le graveur peut disposer, sur deux registres superposés, de petites scènes à échelle réduite. Souvent, l'un des deux petits registres est occupé, en totalité ou partiellement, par le nom du propriétaire du sceau ${ }^{10}$.

(9) Cette figure familière de l'imagerie babylonienne du second millénaire occupe le plus souvent une place très discrète dans la composition. Elle peut nous faire penser à ces images de donateurs qui apparaissent en marge des tableaux religieux de l'Occident médiéval.

(10) On précisera ici que ces inscriptions cunéiformes ne servent que dans de très rares exceptions d'aide à la compréhension de l'image, même lorsqu'elles deviennent des prières aux dieux. Ecriture et image se sont ainsi développées en toute indépendance sur les sceaux du Proche-Orient ancien. 

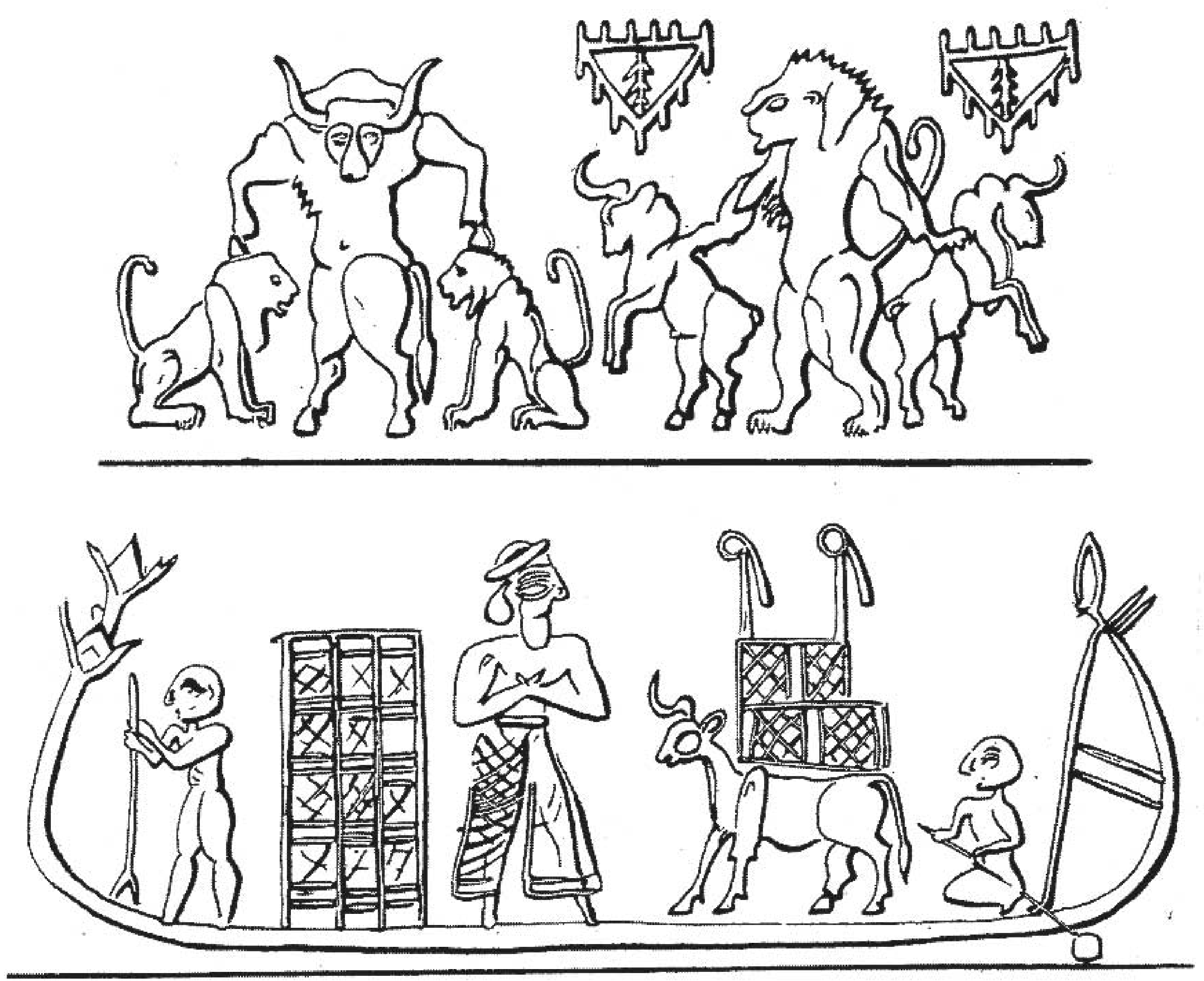

Fig. 7a-b: empreintes d'un cylindre proto-élamite, vers 3000-2800 av. J.-C., et d'un cylindre d'Uruk III, vers 3000 (GMA, $\mathrm{n}^{\circ} 585$ et 655$)$

Ce mode de composition a été très en faveur au second millénaire dans la glyptique syrienne ${ }^{11}$ puis mitannienne et syro-hittite (fig. 9a-b).

C'est dans le domaine des représentations architecturales que les problèmes d'échelle sont souvent les plus sensibles. On sait que l'architecture du Proche-Orient ancien, celle de la Mésopotamie en particulier, a généralement mal supporté l'épreuve du temps en raison de la fragilité du matériau utilisé le plus souvent, à savoir la brique crue, séchée simplement au soleil. Si les plans des murs ou de leurs fondations sont bien retrouvés, il n'en est généralement pas de même pour l'élévation. Proportions, volumes, décors des superstructures échappent souvent à l'archéologue. Aussi se doit-il d'être très attentif à toute image susceptible de garder la mémoire des formes architecturales perdues. En dehors des bas-reliefs assyriens, qui constituent une mine d'informations iconographiques, mais cantonnée à certaines formes architecturales et restreinte dans le temps (IX ${ }^{\mathrm{e}}$-VII ${ }^{\mathrm{e}}$ siècles av. J.-C.), c'est bien l'imagerie des sceaux qui offre ici aussi quelques ressources non négligeables. Certes, les édifices qui ont pu être reproduits sur les sceaux sont surtout des édifices religieux et, d'autre part, les dimensions obligatoirement réduites des images ne permettent pas d'espérer des exemples très fidèles à la réalité ni très détaillés. On comprend en outre aisément que les proportions des bâtiments par rapport aux autres éléments du décor ne pouvaient guère être respectées (fig. 10a-b).

(11) Dans le domaine syrien, la cour 106 du palais amorite de Mari a livré les vestiges d'un décor peint, malheureusement fragmentaire, qui adopte également ce mode particulier de composition, consacré ici à une scène de sacrifice rituel de taureaux. Le roi de Mari y était sans doute représenté face à une divinité: cf. André PArrot, Mission archéologique de Mari II, Le palais, 2, Peintures murales, Paris, Geuthner, 1958, pl. VI et Ba. 

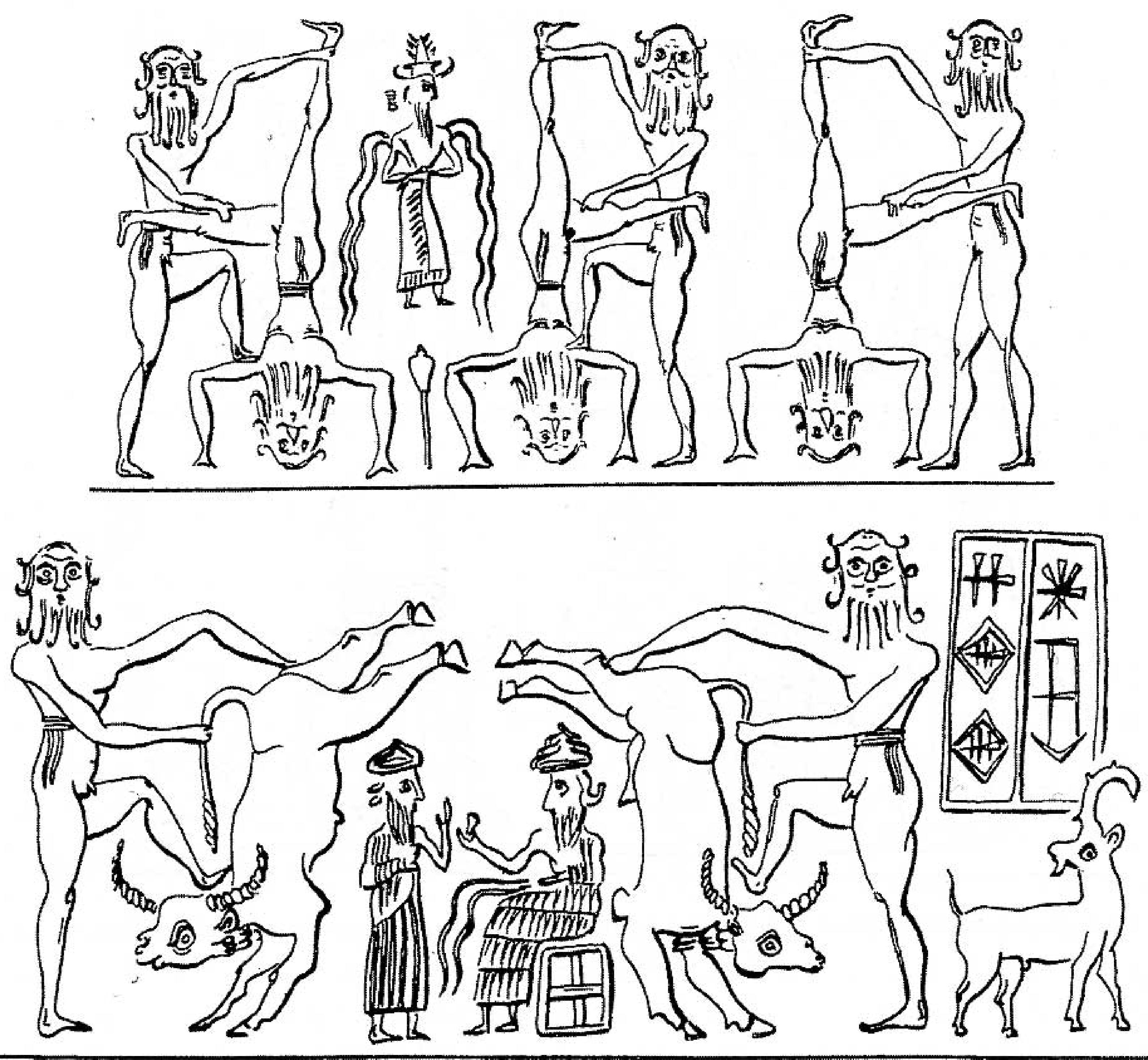

Fig. 8a-b: empreintes de cylindres akkadiens montrant le dieu de l'aps $\hat{u}, \mathrm{Ea}$, et ses acolytes (GMA, $\left.\mathrm{n}^{\circ} 1470-71\right)$

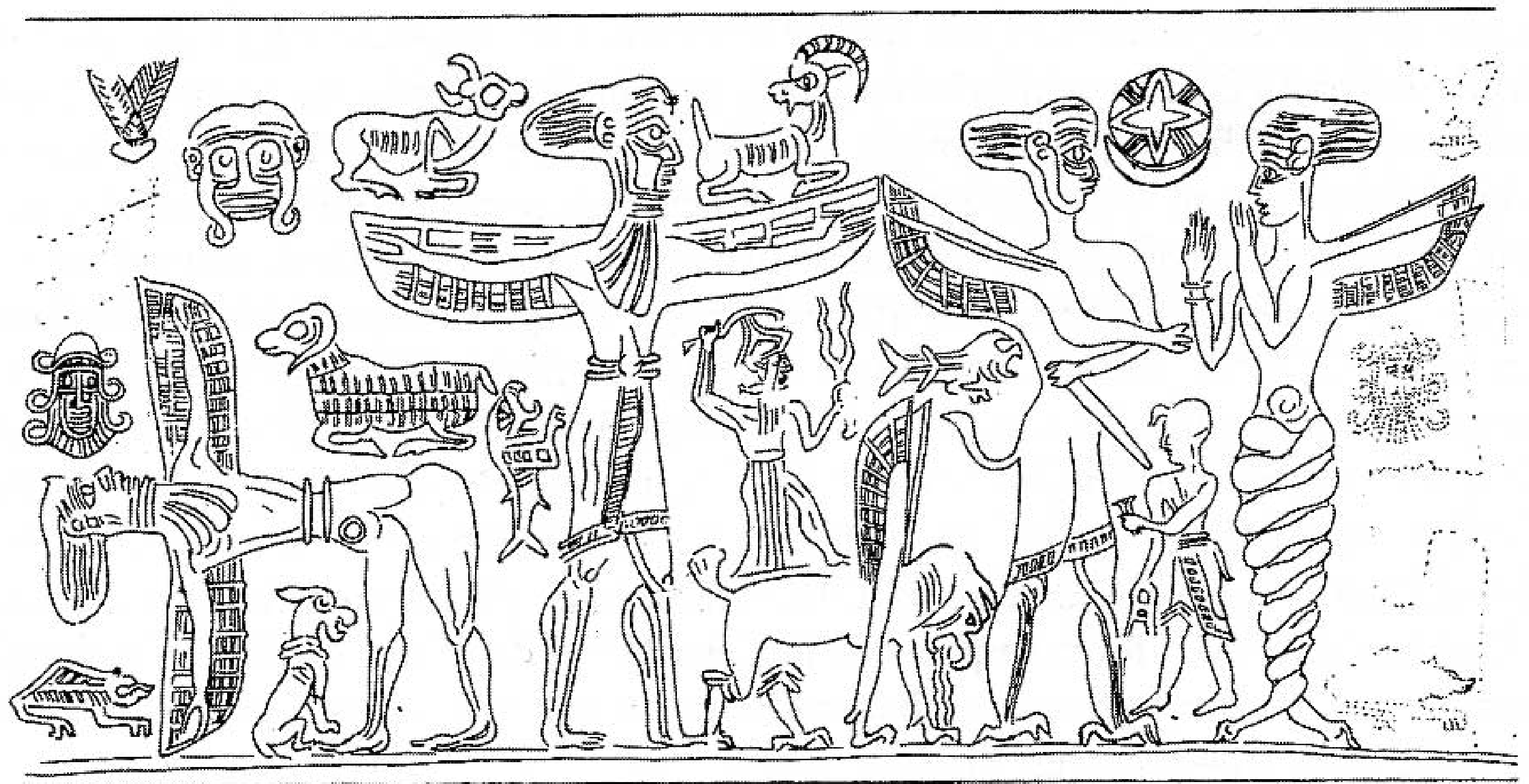

Fig. 8c: empreinte d'un cylindre du British Museum, $1^{\mathrm{e}}$ moitié du $2^{\mathrm{e}}$ millénaire av. J.-C.

(d'après E. PorAdA, «Remarks on Mitannian (Hurrian) and Middle Assyrian Glyptic Art», Akkadica 13, 1979, fig. 3) 

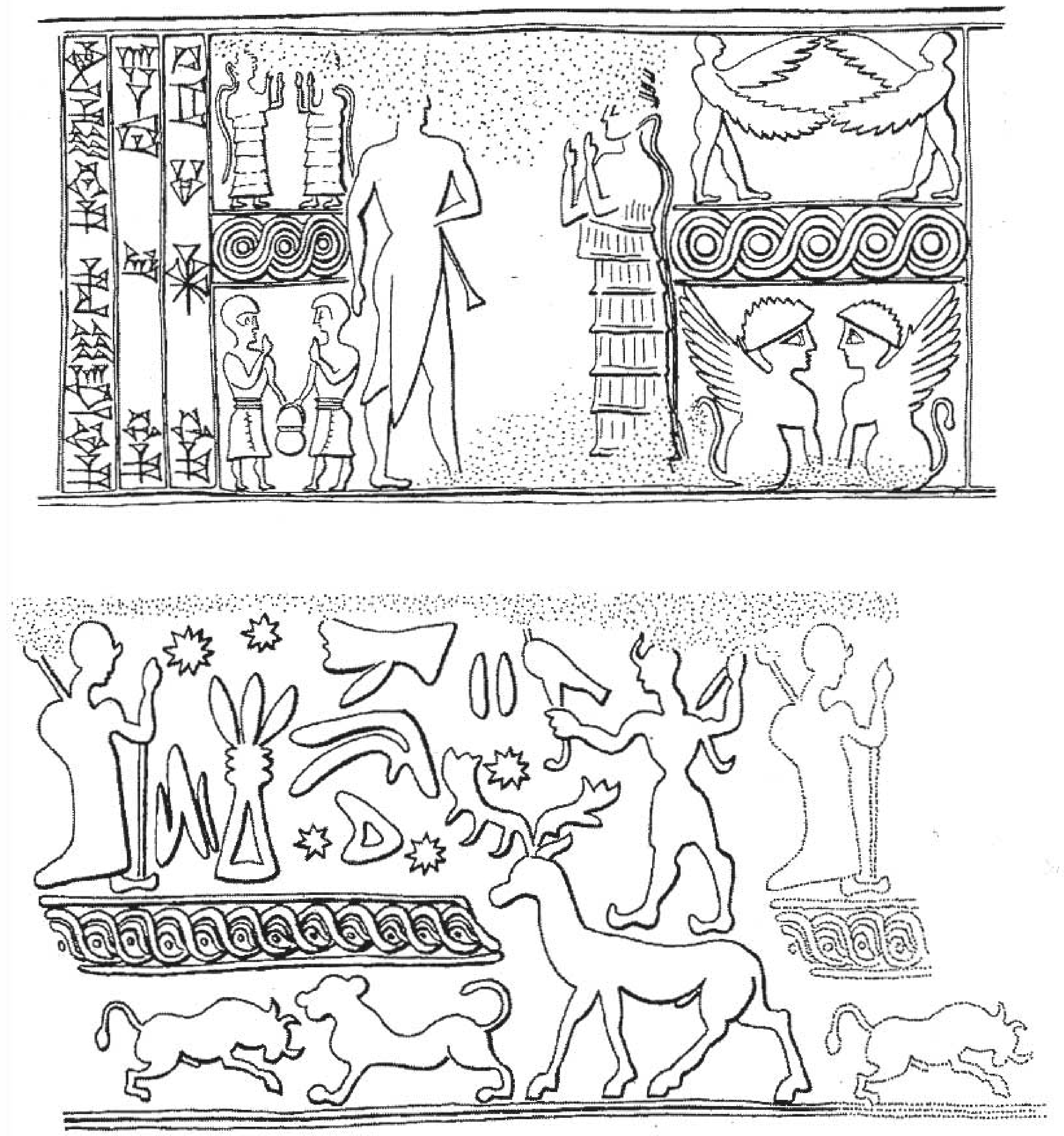

Fig. 9a-b : empreinte du sceau d'un fonctionnaire du roi de Mari, XVIII' siècle av. J.-C. (d'après D. BEYER, «Le sceau de Kabi-Addu, fils d'Asqudum», MARI 3, 1984, p. 356) et d'un sceau-cylindre syro-hittite de Meskéné-Emar, XIII' siècle av. J.-C. (d'après D. BEYER, Emar IV. Les sceaux, OBO, Fribourg, 2001, n A78)

Ainsi, une série particulière de documents, appartenant essentiellement à la période du Dynastique Archaïque, présente-t-elle autant d'exemples d'interprétation problématique, en évoquant le thème de la construction d'un édifice à étages (fig. $11 \mathrm{a}-\mathrm{c})^{12}$. Ce thème, associé souvent à celui, de nature mythologique, du dieu-bateau, illustre une cérémonie présidée normalement par un personnage assis qui peut porter la tiare à cornes des divinités, et qui fait l'objet d'un culte. Il est accompagné par plusieurs personnages qui portent un objet plus ou moins volumineux sur leur tête, évoquant tout aussi bien des ouvriers-maçons que des porteurs d'offrandes. Deux d'entre eux semblent poser un objet de ce type, ou de forme plus carrée, au sommet d'une sorte d'édicule. On a voulu y voir le dessin d'une brique, parfois même d'un dernier étage vu en plan, complétant la construction de l'édicule, considéré alors par certains comme l'image d'une ziggurat, une haute tour à étages, type architectural caractéristique de l'architecture sacrée mésopotamienne, mais qui n'apparaît pas avant la fin du $\mathrm{III}^{\mathrm{e}}$ millénaire. L'aspect de certains de ces édicules, sur des sceaux-cylindres au décor plus schématique, évoque davantage une butte ou une meule de foin qu'une véritable construction! L'échelle appliquée sur

(12) On consultera sur cette question les pages de Pierre Amiet, La glyptique mésopotamienne archaïque, ( $2^{e}$ édition), Paris, CNRS, 1980 , p. 181 et suivantes, et pl. 108-110 et 112. 

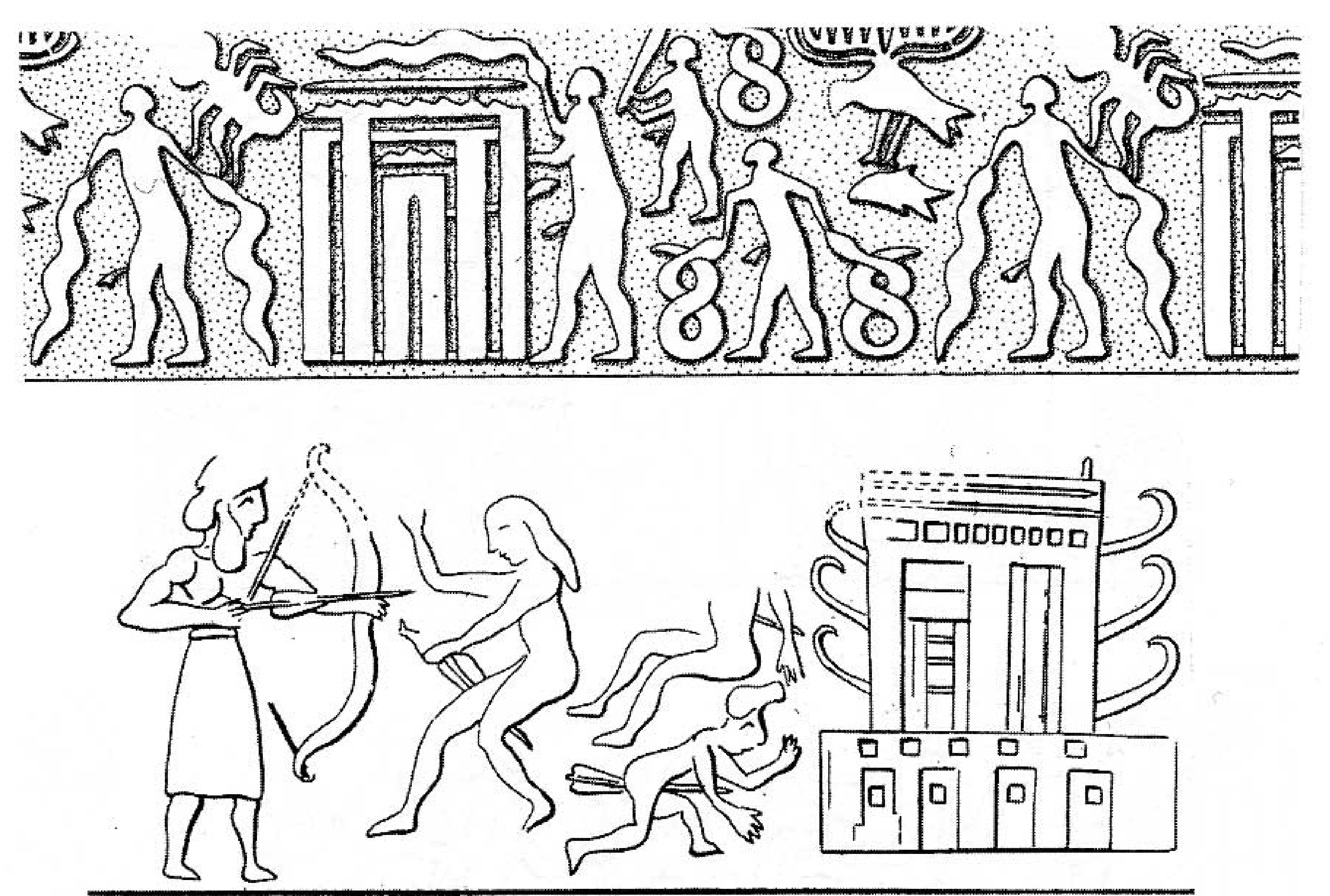

Fig. 10a-b: empreinte d'un sceau-cylindre de la période d'Uruk V, vers 3300: temple et culte du serpent? (d'après R. M. BєHмER, Uruk, Früheste Siegelabrollungen, Ausgrabungen in Uruk-Warka Endberichte 24, Mainz, Philipp von Zabern, 1999, pl. 41, $\mathrm{n}^{\circ}$ 13. T.W); empreinte d'un sceau-cylindre de Suse, vers 3000: le «roi-prêtre» abattant ses ennemis devant la façade d'un temple sur terrasse? (d'après $G M A, \mathrm{n}^{\circ} 659$ )
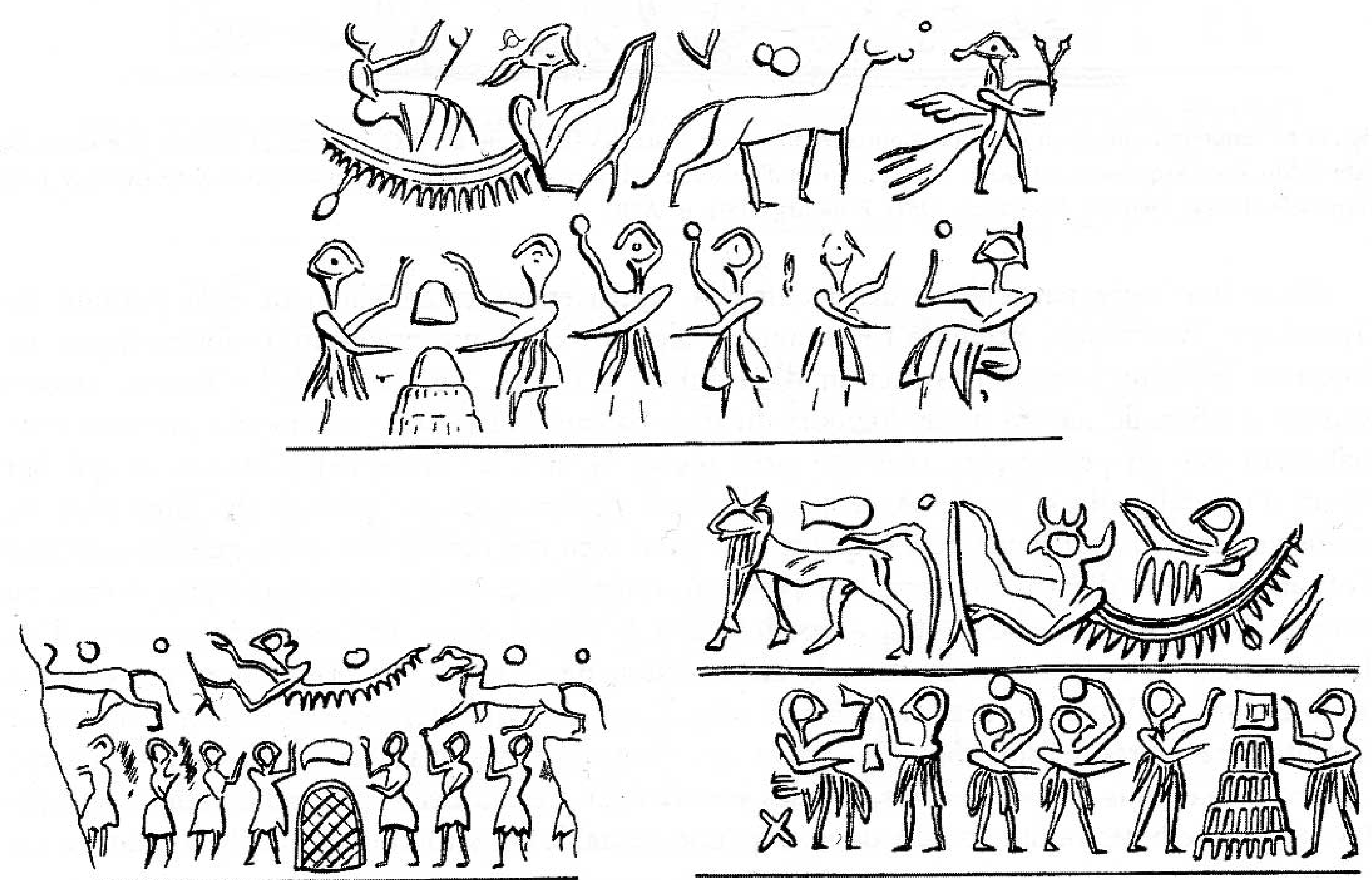

Fig. 11a-c: «dieu-bateau» et construction d'un édifice à étages. Empreintes de sceaux-cylindres du Dynastique Archaïque III de Tell Asmar (a-b) et de Kish (c), (d'après GMA, $\mathrm{n}^{\circ} 1441,1443,1444$ ) 
certains de ces édicules, sur des exemples également très schématiques, évoque bien des constructions élevées, mais qui pourraient tout autant correspondre à des «estrades parfois monumentales, bâties en briques, ou plus souvent, en matériaux périssables (cf. aussi fig. $7 \mathrm{~b}$ ), et jouant le rôle d'autels à escaliers, lointains ancêtres de celui qu'a décrit le prophète Ezéchiel ${ }^{13}$.

On voit bien ici, à propos de cette série thématique, quelles sont les difficultés et les limites de l'interprétation de certaines images d'un répertoire par ailleurs extrêmement précieux pour la connaissance de bien des aspects des civilisations du Proche-Orient ancien.

Dominique BEYER Université Marc Bloch - Strasbourg II

(13) P. Aмret, op. cit., p. 184. On notera que les mêmes difficultés d'interprétation, liées à des questions d'échelle, se posent à propos d'un monument célèbre, unique en son genre au Proche-Orient, le «sît shamshi» de Suse, sorte de maquette en bronze d'une cérémonie en plein air, en l'honneur du lever du soleil d'après la dédicace du roi médio-élamite ShilhakInshushinak, avec la reproduction d'autels à degrés (temples ou ziggurats?) parmi d'autres installations cultuelles, bassins, cuve, pierres dressées et bosquet sacré. On consultera sur ce document le dossier récent paru dans La cité royale de Suse, découvertes archéologiques en Iran conservées au Musée du Louvre, (P. O. HARPER, J. ARUz et F. TALLON, éd)., Paris, RMN, 1994, p. 137-141. 